PHYSICAL REVIEW E 92, 019902(E) (2015)

\title{
Publisher's Note: Stochastic dynamics and logistic population growth \\ [Phys. Rev. E 91, 062133 (2015)]
}

Vicenç Méndez, Michael Assaf, Daniel Campos, and Werner Horsthemke

(Received 2 July 2015; published 8 July 2015)

DOI: 10.1103/PhysRevE.92.019902

PACS number(s): 05.40.-a, 87.23.Cc, 87.10.Mn, 99.10.Fg

This paper was published online on 24 June 2015 with a typographical error in an inline equation after Eq. (4.8) on page 9. The inline equation should read as " $q=\langle n\rangle / N$ ". The inline equation has been corrected as of 26 June 2015 . The inline equation is correct in the printed version of the journal. 